\title{
ITERATED $G$-FRACTIONAL VECTOR REPRESENTATION FORMULAE AND INEQUALITIES FOR BANACH SPACE VALUED FUNCTIONS
}

\begin{abstract}
Here we present very general iterated fractional Bochner integral representation formulae for Banach space valued functions. Based on these we derive generalized and iterated left and right: fractional Poincaré type inequalities, fractional Opial type inequalities and fractional Hilbert-Pachpatte inequalities. All these inequalities are very general having in their background Bochner type integrals.
\end{abstract}

Key words: Banach space valued functions, Iterated vector generalized fractional derivative, Caputo fractional derivative, Iterated generalized vector fractional integral inequalities, Bochner integral, fractional vector representation formulae.

2010 Mathematical Subject Classification: 26A33, 26B40, 26D10, 26D15, 46B25, 46E40.

\section{Background. We need}

Definition 1. [2] Let $[a, b] \subset \mathbb{R},(X,\|\cdot\|)$ a Banach space, $g \in C^{1}([a, b])$ and increasing, $f \in C([a, b], X), \nu>0$.

We define the left Riemann-Liouville generalized fractional Bochner integral operator

$$
\left(I_{a+; g}^{\nu} f\right)(x):=\frac{1}{\Gamma(\nu)} \int_{a}^{x}(g(x)-g(z))^{\nu-1} g^{\prime}(z) f(z) d z,
$$

$\forall x \in[a, b]$, where $\Gamma$ is the gamma function.

The last integral is of Bochner type. Since $f \in C([a, b], X)$, then $f \in L_{\infty}([a, b], X)$. By [2] we get that $I_{a+; g}^{\nu} f \in C([a, b], X)$. Above we set $I_{a+; g}^{0} f:=f$ and see that $\left(I_{a+; g}^{\nu} f\right)(a)=0$.

(C) Petrozavodsk State University, 2020 
When $g$ is the identity function $i d$, we get that $I_{a+; i d}^{\nu}=I_{a+}^{\nu}$, the ordinary left Riemann-Liouville fractional integral

$$
\left(I_{a+}^{\nu} f\right)(x)=\frac{1}{\Gamma(\nu)} \int_{a}^{x}(x-t)^{\nu-1} f(t) d t,
$$

$\forall x \in[a, b],\left(I_{a+}^{\nu} f\right)(a)=0$.

We need

Theorem 1. [2] Let $\mu, \nu>0$ and $f \in C([a, b], X)$. Then

$$
I_{a+; g}^{\mu} I_{a+; g}^{\nu} f=I_{a+; g}^{\mu+\nu} f=I_{a+; g}^{\nu} I_{a+;}^{\mu} f .
$$

We need

Definition 2. [2] Let $[a, b] \subset \mathbb{R},(X,\|\cdot\|)$ a Banach space, $g \in C^{1}([a, b])$ and increasing, $f \in C([a, b], X), \nu>0$.

We define the right Riemann-Liouville generalized fractional Bochner integral operator

$$
\left(I_{b-; g}^{\nu} f\right)(x):=\frac{1}{\Gamma(\nu)} \int_{x}^{b}(g(z)-g(x))^{\nu-1} g^{\prime}(z) f(z) d z,
$$

$\forall x \in[a, b]$, where $\Gamma$ is the gamma function.

The last integral is of Bochner type. Since $f \in C([a, b], X)$, then $f \in L_{\infty}([a, b], X)$. By [2] we get that $I_{b-; g}^{\nu} f \in C([a, b], X)$. Above we set $I_{b-; g}^{0} f:=f$ and see that $\left(I_{b-; g}^{\nu} f\right)(b)=0$.

When $g$ is the identity function $i d$, we get that $I_{b-; i d}^{\nu}=I_{b-}^{\nu}$, the ordinary right Riemann-Liouville fractional integral

$$
\left(I_{b-}^{\nu} f\right)(x)=\frac{1}{\Gamma(\nu)} \int_{x}^{b}(t-x)^{\nu-1} f(t) d t,
$$

$\forall x \in[a, b]$, with $\left(I_{b-}^{\nu} f\right)(b)=0$.

We need

Theorem 2. [2] Let $\mu, \nu>0$ and $f \in C([a, b], X)$. Then

$$
I_{b-;}^{\mu} I_{b-; g}^{\nu} f=I_{b-; g}^{\mu+\nu} f=I_{b-; g}^{\nu} I_{b-; g}^{\mu} f .
$$


We will use

Definition 3. [2] Let $\alpha>0,\lceil\alpha\rceil=n,\lceil\cdot\rceil$ the ceiling of the number. Let $f \in C^{n}([a, b], X)$, where $[a, b] \subset \mathbb{R}$, and $(X,\|\cdot\|)$ is a Banach space. Let $g \in C^{1}([a, b])$, strictly increasing, such that $g^{-1} \in C^{n} t([g(a), g(b)])$.

We define the left generalized $g$-fractional derivative $X$-valued of $f$ of order $\alpha$ as follows:

$$
\left(D_{a+; g}^{\alpha} f\right)(x):=\frac{1}{\Gamma(n-\alpha)} \int_{a}^{x}(g(x)-g(t))^{n-\alpha-1} g^{\prime}(t)\left(f \circ g^{-1}\right)^{(n)}(g(t)) d t,
$$

$\forall x \in[a, b]$. The last integral is of Bochner type.

If $\alpha \notin \mathbb{N}$, by [2], we have that $\left(D_{a+; g}^{\alpha} f\right) \in C([a, b], X)$.

We see that

$$
\left(I_{a+; g}^{n-\alpha}\left(\left(f \circ g^{-1}\right)^{(n)} \circ g\right)\right)(x)=\left(D_{a+; g}^{\alpha} f\right)(x), \quad \forall x \in[a, b] .
$$

We set

$$
\begin{aligned}
D_{a+; g}^{n} f(x):= & \left(\left(f \circ g^{-1}\right)^{(n)} \circ g\right)(x) \in C([a, b], X), \quad n \in \mathbb{N}, \\
& D_{a+; g}^{0} f(x)=f(x), \quad \forall x \in[a, b] .
\end{aligned}
$$

When $g=i d$, then

$$
D_{a+; g}^{\alpha} f=D_{a+; i d}^{\alpha} f=D_{* a}^{\alpha} f,
$$

the usual left $X$-valued Caputo fractional derivative, see [3].

We will use

Definition 4. [2] Let $\alpha>0,\lceil\alpha\rceil=n,\lceil\cdot\rceil$ the ceiling of the number. Let $f \in C^{n}([a, b], X)$, where $[a, b] \subset \mathbb{R}$, and $(X,\|\cdot\|)$ is a Banach space. Let $g \in C^{1}([a, b])$, strictly increasing, such that $g^{-1} \in C^{n}([g(a), g(b)])$.

We define the right generalized $g$-fractional derivative $X$-valued of $f$ of order $\alpha$ as follows:

$$
\left(D_{b-; g}^{\alpha} f\right)(x):=\frac{(-1)^{n}}{\Gamma(n-\alpha)} \int_{x}^{b}(g(t)-g(x))^{n-\alpha-1} g^{\prime}(t)\left(f \circ g^{-1}\right)^{(n)}(g(t)) d t,
$$

$\forall x \in[a, b]$. The last integral is of Bochner type.

If $\alpha \notin \mathbb{N}$, by [2], we have that $\left(D_{b-; g}^{\alpha} f\right) \in C([a, b], X)$. 
We see that

$$
I_{b-; g}^{n-\alpha}\left((-1)^{n}\left(f \circ g^{-1}\right)^{(n)} \circ g\right)(x)=\left(D_{b-; g}^{\alpha} f\right)(x), a \leqslant x \leqslant b .
$$

We set

$$
\begin{gathered}
D_{b-; g}^{n} f(x):=(-1)^{n}\left(\left(f \circ g^{-1}\right)^{n} \circ g\right)(x) \in C([a, b], X), n \in \mathbb{N}, \\
D_{b-; g}^{0} f(x):=f(x), \quad \forall x \in[a, b] .
\end{gathered}
$$

When $g=i d$, then

$$
D_{b-; g}^{\alpha} f(x)=D_{b-; i d}^{\alpha} f(x)=D_{b-}^{\alpha} f,
$$

the usual right $X$-valued Caputo fractional derivative, see [3].

We make

Remark 1. All as in Definition 3. We have (by Theorem 2.5, p. 7, [5])

$$
\begin{gathered}
\left\|\left(D_{a+; g}^{\alpha} f\right)(x)\right\| \leqslant \frac{1}{\Gamma(n-\alpha)} \int_{a}^{x}(g(x)-g(t))^{n-\alpha-1} g^{\prime}(t)\left\|\left(f \circ g^{-1}\right)^{(n)}(g(t))\right\| d t \\
\leqslant \frac{\left.\left\|\left(f \circ g^{-1}\right)^{(n)} \circ g\right\|_{\infty,[a, b]} \int_{g(a)}^{g(x)}(g x)-g(t)\right)^{n-\alpha-1} d g(t)=}{\Gamma(n-\alpha)} \\
=\frac{\left\|\left(f \circ g^{-1}\right)^{(n)} \circ g\right\|_{\infty,[a, b]}}{\Gamma(n-\alpha+1)}(g(x)-g(a))^{n-\alpha} .
\end{gathered}
$$

That is

$$
\left\|\left(D_{a+; g}^{\alpha} f\right)(x)\right\| \leqslant \frac{\left\|\left(f \circ g^{-1}\right)^{(n)} \circ g\right\|_{\infty,[a, b]}}{\Gamma(n-\alpha+1)}(g(x)-g(a))^{n-\alpha},
$$

$\forall x \in[a, b]$.

If $\alpha \notin \mathbb{N}$, then $\left(D_{a+; g}^{\alpha} f\right)(a)=0$.

Similarly, by Definition 4 we derive

$$
\left\|\left(D_{b-; g}^{\alpha} f\right)(x)\right\| \leqslant \frac{1}{\Gamma(n-\alpha)} \int_{x}^{b}(g(t)-g(x))^{n-\alpha-1} g^{\prime}(t)\left\|\left(f \circ g^{-1}\right)^{(n)}(g(t))\right\| d t \leqslant
$$




$$
\begin{aligned}
& \leqslant \frac{\left\|\left(f \circ g^{-1}\right)^{(n)} \circ g\right\|_{\infty,[a, b]}}{\Gamma(n-\alpha} \int_{g(x)}^{g(b)}(g(t)-g(x))^{n-\alpha-1} d g(t)= \\
& \quad=\frac{\left\|\left(f \circ g^{-1}\right)^{(n)} \circ g\right\|_{\infty,[a, b]}}{\Gamma(n-\alpha+1)}(g(b)-g(x))^{n-\alpha} .
\end{aligned}
$$

That is

$$
\left\|\left(D_{b-; g}^{\alpha} f\right)(x)\right\| \leqslant \frac{\left\|\left(f \circ g^{-1}\right)^{(n)} \circ g\right\|_{\infty,[a, b]}}{\Gamma(n-\alpha+1)}(g(b)-g(x))^{n-\alpha},
$$

$\forall x \in[a, b]$.

If $\alpha \notin \mathbb{N}$, then $\left(D_{b-; g}^{\alpha} f\right)(b)=0$.

Notation 3 We denote by

$$
\begin{aligned}
D_{a+; g}^{n \alpha}:=D_{a+; g}^{\alpha} D_{a+; g}^{\alpha} \ldots D_{a+; g}^{\alpha} \quad \text { (n times) }, n \in \mathbb{N} & \\
I_{a+; g}^{n \alpha}: & =I_{a+; g}^{\alpha} I_{a+; g}^{\alpha} \ldots I_{a+; g}^{\alpha} \\
D_{b-; g}^{n \alpha} & :=D_{b-; g}^{\alpha} D_{b-; g}^{\alpha} \ldots D_{b-; g}^{\alpha},
\end{aligned}
$$

and

$$
I_{b-; g}^{n \alpha}:=I_{b-; g}^{\alpha} I_{b-; g}^{\alpha} \ldots I_{b-; g}^{\alpha}
$$

( $n$ times), $n \in \mathbb{N}$.

We are motivated by the following generalized fractional Ostrowski type inequality:

Theorem 4. [2] Let $g \in C^{1}([a, b])$ and strictly increasing, such that $g^{-1} \in C^{1}([g(a), g(b)])$, and $0<\alpha<1, n \in \mathbb{N}, f \in C^{1}([a, t], X)$, where $(X,\|\cdot\|)$ is a Banach space. Let $x_{0} \in[a, b]$ be fixed. Assume that $F_{k}^{x_{0}}:=D_{x_{0}-; g}^{k \alpha} f$, for $k=1, \ldots, n$, fulfill $F_{k}^{x_{0}} \in C^{1}\left(\left[a, x_{0}\right], X\right)$ and

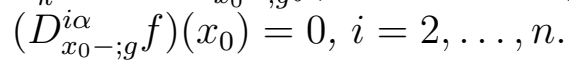

Similarly, we assume that $G_{k}^{x_{0}}:=D_{x_{0}+; g}^{k \alpha} f$, for $k=1, \ldots, n$, fulfill $G_{k}^{x_{0}} \in C^{1}\left(\left[x_{0}, b\right], X\right)$ and $\left(D_{x_{0}+; g}^{i \alpha} f\right)\left(x_{0}\right)=0, i=2, \ldots, n$.

Then

$$
\left\|\frac{1}{b-a} \int_{a}^{b} f(x) d x-f\left(x_{0}\right)\right\| \leqslant \frac{1}{(b-a) \Gamma((n+1) \alpha+1)} \times
$$




$$
\begin{aligned}
& \times\left\{\left(g(b)-g\left(x_{0}\right)\right)^{(n+1) \alpha}\left(b-x_{0}\right)\left\|D_{x_{0}+; g}^{(n+1) \alpha} f\right\|_{\infty,\left[x_{0}, b\right]}+\right. \\
& \left.+\left(g\left(x_{0}\right)-g(a)\right)^{(n+1) \alpha}\left(x_{0}-a\right)\left\|D_{x_{0}-; g}^{(n+1) \alpha} f\right\|_{\infty,\left[a, x_{0}\right]}\right\} .
\end{aligned}
$$

In this work we will present several generalized fractional Bochner integral inequalities.

We mention the following $g$-left generalized modified $X$-valued Taylor's formula.

Theorem 5. [2] Let $0<\alpha \leqslant 1, n \in \mathbb{N}, f \in C^{1}([a, b], X), g \in C^{1}([a, b])$, strictly increasing, such that $g^{-1} \in C^{1}([g(a), g(b)])$. Let $F_{k}:=D_{a+; g}^{k \alpha} f$, $k=1, \ldots, n$, that fulfill $F_{k} \in C^{1}([a, b], X)$.

Then

$$
\begin{gathered}
f(x)=\sum_{i=0}^{n} \frac{(g(x)-g(a))^{i \alpha}}{\Gamma(i \alpha+1)}\left(D_{a+; g}^{i \alpha} f\right)(a)+ \\
+\frac{1}{\Gamma((n+1) \alpha)} \int_{a}^{x}(g(x)-g(t))^{(n+1) \alpha-1} g^{\prime}(t)\left(D_{a+; g}^{(n+1) \alpha} f\right)(t) d t
\end{gathered}
$$

$\forall x \in[a, b]$.

Clearly here $D_{a+; g}^{(n+1) \alpha} f \in C([a, b], X)$.

We also mention the following $g$-right generalized modified $X$-valued Taylor's formula.

Theorem 6. [2] Let $f \in C^{1}([a, b], X), g \in C^{1}([a, b])$, strictly increasing, such that $g^{-1} \in C^{1}([g(a), g(b)])$. Suppose that $F_{k}:=D_{b-; g}^{k \alpha} f, k=1, \ldots, n$, fulfill $F_{k} \in C^{1}([a, b], X)$, where $0<\alpha \leqslant 1, n \in \mathbb{N}$.

Then

$$
\begin{gathered}
f(x)=\sum_{i=0}^{n} \frac{(g(b)-g(x))^{i \alpha}}{\Gamma(i \alpha+1)}\left(D_{b-; g}^{i \alpha} f\right)(b)+ \\
+\frac{1}{\Gamma((n+1) \alpha)} \int_{x}^{b}(g(t)-g(x))^{(n+1) \alpha-1} g^{\prime}(t)\left(D_{b-; g}^{(n+1) \alpha} f\right)(t) d t
\end{gathered}
$$

$\forall x \in[a, b]$.

Clearly here $D_{b-; g}^{(n+1) \alpha} f \in C([a, b], X)$. 
For differentiation of functions from real numbers to normed linear spaces the definition is the same as for the real valued functions, however the limit and convergence is in the norm of linear space $(X,\|\cdot\|)$.

We state

Corollary 1. (to Theorem 5) Let $0<\alpha<1, n \in \mathbb{N}, f \in C^{1}([a, b], X)$, $g \in C^{1}([a, b])$, strictly increasing, such that $g^{-1} \in C^{1}([g(a), g(b)])$. Assume $D_{a+; g}^{k \alpha} f \in C^{1}([a, b], X), k=1, \ldots, n$, and $\left(D_{a+; g}^{i \alpha} f\right)(a)=0, i=0,2,3, \ldots, n$.

Then

$$
f(x)=\frac{1}{\Gamma((n+1) \alpha)} \int_{a}^{x}(g(x)-g(t))^{(n+1) \alpha-1} g^{\prime}(t)\left(D_{a+; g}^{(n+1) \alpha} f\right)(t) d t,
$$

$\forall x \in[a, b]$.

We state

Corollary 2. (to Theorem 6) Let $f \in C^{1}([a, b], X), g \in C^{1}([a, b])$, strictly increasing, such that $g^{-1} \in C^{1}([g(a), g(b)]$. Suppose that for $k=1, \ldots, n$ $D_{b-; g}^{k \alpha} f \in C^{1}([a, b], X)$, where $0<\alpha<1, n \in \mathbb{N}$. We further assume that $\left(D_{b-; g}^{i \alpha} f\right)(b)=0, i=0,2,3, \ldots, n$.

Then

$$
f(x)=\frac{1}{\Gamma((n+1) \alpha)} \int_{x}^{b}(g(t)-g(x))^{(n+1) \alpha-1} g^{\prime}(t)\left(D_{b-; g}^{(n+1) \alpha} f\right)(t) d t,
$$

$\forall x \in[a, b]$.

For the Bochner integral excellent resources are [1, pp. 422-428], [4] and [7].

2. Main Results. We give the following representation formula:

Theorem 7. All as in Corollary 1. Let $\gamma>0$ with $\lceil\gamma\rceil=m<n+1$, such that $m<(n+1) \alpha($ i. e. $m / n+1<\alpha<1)$. Then

$$
\begin{gathered}
\left(D_{a+; g}^{\gamma} f\right)(x)= \\
\left.=\frac{1}{\Gamma((n+1) \alpha-\gamma)} \int_{a}^{x}(g(x)-g(t))^{(n+1) \alpha-\gamma-1} g^{\prime}(t)\left(D_{a+; g}^{(n+1) \alpha} f\right) t\right) d t
\end{gathered}
$$


$\forall x \in[a, b]$ and $\left(D_{a+; g}^{\gamma} f\right) \in C([a, b], X)$.

Proof. By Corollary 1 we have

$f(x)=\frac{1}{\Gamma((n+1) \alpha)} \int_{a}^{x}(g(x)-g(t))^{(n+1) \alpha-1} g^{\prime}(t)\left(D_{a+; g}^{(n+1) \alpha} f\right)(t) d t, \forall x \in[a, b]$.

We can write

$$
f(x)=\frac{1}{\Gamma((n+1) \alpha)} \int_{a}^{x}(g(x)-g(t))^{(n+1) \alpha-1}\left(D_{a+; g}^{(n+1) \alpha} f\right)(t) d g(t)=
$$

(set $z:=g(t), a \leqslant t \leqslant b$ )

$=\frac{1}{\Gamma((n+1) \alpha)} \int_{g(a)}^{g(x)}(g(x)-z)^{(n+1) \alpha-1}\left(\left(D_{a+; g}^{(n+1) \alpha} f\right) \circ g^{-1}\right)(z) d z, \quad \forall x \in[a, b]$.

Hence it holds $(y=g(x))$

$$
\left(f \circ g^{-1}\right)(y)=\frac{1}{\Gamma((n+1) \alpha)} \int_{g(a)}^{y}(y-z)^{(n+1) \alpha-1}\left(\left(D_{a+; g}^{(n+1) \alpha} f\right) \circ g^{-1}\right)(z) d z,
$$

$\forall y \in[g(a), g(b)]$.

By assuming $(n+1) \alpha-1>0$ (equivalently, $\left.\alpha>\frac{1}{n+1}\right)$ and by [2], we get:

$$
\left(\left(f \circ g^{-1}\right)(y)\right)^{\prime}=\frac{(n+1) \alpha-1}{\Gamma((n+1) \alpha)} \int_{g(a)}^{y}(y-z)^{(n+1) \alpha-2}\left(\left(D_{a+; g}^{(n+1) \alpha} f\right) \circ g^{-1}\right)(z) d z,
$$

$\forall y \in[g(a), g(b)]$.

If $(n+1) \alpha-2>0$ (equivalently, $\alpha>2 / n+1$ ), we get

$$
\begin{gathered}
\left(\left(f \circ g^{-1}\right)(y)\right)^{\prime \prime}=\frac{((n+1) \alpha-1)((n+1) \alpha-2)}{\Gamma((n+1) \alpha)} \times \\
\times \int_{g(a)}^{y}(y-z)^{(n+1) \alpha-3}\left(\left(D_{a+; g}^{(n+1) \alpha} f\right) \circ g^{-1}\right)(z) d z, \quad \forall y \in[g(a), g(b)] .
\end{gathered}
$$


In general, if $(n+1) \alpha-m>0$ (equivalently, $\alpha>m / n+1)$ we get that there exists

$$
\begin{gathered}
\left(f \circ g^{-1}\right)^{(m)}(y)=\frac{\prod_{j=1}^{m}((n+1) \alpha-j)}{\Gamma((n+1) \alpha)} \times \\
\times \int_{g(a)}^{y}(y-z)^{(n+1) \alpha-m-1}\left(\left(D_{a+; g}^{(n+1) \alpha} f\right) \circ g^{-1}\right)(z) d z, \quad \forall y \in[g(a), g(b)] .
\end{gathered}
$$

By [2] we get $\left(f \circ g^{-1}\right)^{(m)} \in C([g(a), g(b)])$.

By (1) we have that

$$
\begin{gathered}
\left(f \circ g^{-1}\right)^{(m)}(y)=\frac{\prod_{j=1}^{m}((n+1) \alpha-j) \Gamma((n+1) \alpha-m)}{\Gamma((n+1) \alpha)} \times \\
\times I_{g(a)+}^{(n+1) \alpha-m}\left(\left(D_{a+; g}^{(n+1) \alpha} f\right) \circ g^{-1}\right)(y)=I_{g(a)+}^{(n+1) \alpha-m}\left(\left(D_{a+;}^{(n+1) \alpha} f\right) \circ g^{-1}\right)(y) .
\end{gathered}
$$

That is

$$
\left(f \circ g^{-1}\right)^{(m)}(y)=I_{g(a)+}^{(n+1) \alpha-m}\left(\left(D_{a+; g}^{(n+1) \alpha} f\right) \circ g^{-1}\right)(y), \quad \forall y \in[g(a), g(b)],
$$

and

$$
\left(f g^{-1}\right)^{(m)}(g(x))=I_{g(a)+}^{(n+1) \alpha-m}\left(\left(D_{a+; g}^{(n+1) \alpha} f\right) \circ g^{-1}\right)(g(x)), \quad \forall x \in[a, b] .
$$

Clearly, it holds (see [6], Lemma B.4.10, and [7], p. 158)

$$
\left(f \circ g^{-1}\right)^{(m)}(g(x))=\left(I_{a+; g}^{(n+1) \alpha-m}\left(D_{a+; g}^{(n+1) \alpha} f\right)\right)(x), \quad \forall x \in[a, b] .
$$

Let $\gamma>0$ with $\lceil\gamma\rceil=m<n+1$, such that $m<(n+1) \alpha$ (equivalently, $\alpha>m / n+1)$.

We have that (case of $\gamma<m$ )

$$
\begin{gathered}
\left(D_{a+; g}^{\gamma} f\right)(x) \stackrel{(3)}{=}\left(I_{a+; g}^{m-\gamma}\left(\left(f \circ g^{-1}\right)^{(m)} \circ g\right)\right)(x) \stackrel{(5)}{=} \\
=I_{a+; g}^{m-\gamma}\left(I_{a+; g}^{(n+1) \alpha-m}\left(D_{a+; g}^{(n+1) \alpha} f\right)\right)(x)=\left(I_{a+}^{(n+1) \alpha-\gamma}\left(D_{a+; g}^{(n+1) \alpha} f\right)\right)(x),
\end{gathered}
$$

(by (2)). 
We have proved that

$$
\left(D_{a+; g}^{\gamma} f\right)(x)=\left(I_{a+}^{(n+1) \alpha-\gamma}\left(D_{a+; g}^{(n+1) \alpha} f\right)\right)(x), \quad \forall x \in[a, b],
$$

which is continuous, by [2].

We continue with

Theorem 8. All as in Corollary 1 and let $\gamma>0$ with $\lceil\gamma\rceil=m$. When $\frac{\gamma+m}{n+1}<\alpha<1$, we get that

$$
\begin{gathered}
\left(D_{a+; g}^{2 \gamma} f\right)(x)=\frac{1}{\Gamma((n+1) \alpha-2 \gamma)} \times \\
\times \int_{a}^{x}(g(x)-g(t))^{(n+1) \alpha-2 \gamma-1} g^{\prime}(t)\left(D_{a+; g}^{(n+1) \alpha} f\right)(t) d t,
\end{gathered}
$$

$\forall x \in[a, b]$ and $\left(D_{a+; g}^{2 \gamma} f\right) \in C([a, b], X)$.

Proof. Call $\lambda:=(n+1) \alpha-\gamma-1$, i.e. $\lambda+1=(n+1) \alpha-\gamma$, and call $\delta:=(n+1) \alpha$. Then we can rewrite (4) as

$$
\left(D_{a+; g}^{\gamma} f\right)(x)=\frac{1}{\Gamma(\lambda+1)} \int_{a}^{x}(g(x) g(t))^{\lambda} g^{\prime}(t)\left(D_{a+; g}^{\delta} f\right)(t) d t, \quad \forall x \in[a, b] .
$$

That is $(z=g(t), a \leqslant t \leqslant b)$

$$
\left(D_{a+; g}^{\gamma} f\right)(x)=\frac{1}{\Gamma(\lambda+1)} \int_{g(a)}^{g(x)}(g(x)-z)^{\lambda}\left(\left(D_{a+; g}^{\delta} f\right) \circ g^{-1}\right)(z) d z, \forall x \in[a, b] .
$$

Hence it holds $(y=g(x))$

$$
\left(\left(D_{a+; g}^{\gamma} f\right) \circ g^{-1}\right)(y)=\frac{1}{\Gamma(\lambda+1)} \int_{g(a)}^{y}(y-z)^{\lambda}\left(\left(D_{a+; g}^{\delta} f\right) \circ g^{-1}\right)(z) d z,
$$

$\forall y \in[g(a), g(b)]$.

If $\lambda>0$, then

$$
\left(\left(D_{a+; g}^{\gamma} f\right) \circ g^{-1}\right)^{\prime}(y)=\frac{\lambda}{\Gamma(\lambda+1)} \int_{g(a)}^{y}(y-z)^{\lambda-1}\left(\left(D_{a+; g}^{\delta} f\right) \circ g^{-1}\right)(z) d z
$$


$\forall y \in[g(a), g(b)]$.

If $\lambda-1>0$, then

$$
\left(\left(D_{a+; g}^{\gamma} f\right) \circ g^{-1}\right)^{\prime \prime}(y)=\frac{\lambda(\lambda-1)}{\Gamma t(\lambda+1)} \int_{g(a)}^{y}(y-z)^{\lambda-2}\left(\left(D_{a+; g}^{\delta} f\right) \circ g^{-1}\right)(z) d z
$$

$\forall y \in[g(a), g(b)]$.

If $\lambda-2>0$, then

$$
\left(\left(D_{a+; g}^{\gamma} f\right) \circ g^{-1}\right)^{\prime \prime \prime}(y)=\frac{\lambda(\lambda-1)(\lambda-2)}{\Gamma(\lambda+1)} \int_{g(a)}^{y}(y-z)^{\lambda-3}\left(\left(D_{a+; g}^{\delta} f\right) \circ g^{-1}\right)(z) d z
$$

$\forall y \in[g(a), g(b)]$, etc.

In general, if $\lambda-m+1>0$, then

$$
\begin{aligned}
& \left(\left(D_{a+; g}^{\gamma} f\right) \circ g^{-1}\right)^{(m)}(y)=\frac{\lambda(\lambda-1)(\lambda-2) \ldots(\lambda-m+1)}{\Gamma(\lambda+1)} \times \\
& \times \int_{g(a)}^{y}(y-z)^{(\lambda-m+1)-1}\left(\left(D_{a+; g}^{\delta} f\right) \circ g^{-1}\right)(z) d z= \\
& =\frac{\lambda(\lambda-1)(\lambda-2) \ldots(\lambda-m+1) I_{g(\alpha)+}^{(\lambda-m+1)}\left(\left(D_{a+; g}^{\delta} f\right) \circ g^{-1}\right)(y)}{\Gamma(\lambda+1)}= \\
& =I_{g(a)+}^{(\lambda-m+1)}\left(\left(D_{a+; g}^{\delta} f\right) \circ g^{-1}\right)(y), \quad \forall y \in[g(a), g(b)] .
\end{aligned}
$$

That is, if $\lambda-m+1>0$, we have

$$
\left(\left(D_{a+; g}^{\gamma} f\right) \circ g^{-1}\right)^{(m)}(y)=I_{g(a)+}^{(\lambda-m+1)}\left(\left(D_{a+; g}^{\delta} f\right) \circ g^{-1}\right)(y), \forall y \in[g(a), g(b)] .
$$

Hence

$$
\left(\left(D_{a+; g}^{\gamma} f\right) \circ g^{-1}\right)^{(m)}\left(g(x)=I_{g(a)+}^{(\lambda-m+1)}\left(\left(D_{a+; g}^{(n+1) \alpha} f\right) \circ g^{-1}\right)(g(x)), \forall x \in[a, b] .\right.
$$

Clearly, it holds by change of variables

$$
\left(\left(D_{a+; g}^{\gamma} f\right) \circ g^{-1}\right)^{(m)}(g(x))=\left(I_{a+}^{(\lambda-m+1)}\left(D_{a+; g}^{(n+1) \alpha} f\right)\right)(x),
$$

$\forall x \in[a, b]$, under $\lambda-m+1>0$. 
We see that

$$
\begin{aligned}
&\left(D_{a+; g}^{2 \gamma} f\right)(x)=\left(D_{a+; g}^{\gamma}\left(D_{a+; g}^{\gamma} f\right)\right)(x)= \\
&=\left(I_{a+; g}^{m-\gamma}\left(\left(\left(D_{a+; g}^{\gamma} f\right) \circ g^{-1}\right)^{(m)} \circ g\right)\right)(x) \stackrel{(6)}{=} \\
& \stackrel{(6)}{=}\left(I_{a+; g}^{m-\gamma}\left(I_{a+; g}^{(\lambda-m+1)}\left(D_{a+; g}^{(n+1) \alpha} f\right)\right)\right)(x)= \\
&=\left(I_{a+; g}^{\lambda-\gamma+1}\left(D_{a+; g}^{(n+1) \alpha} f\right)\right)(x)=\left(I_{a+; g}^{(n+1) \alpha-\gamma-1-\gamma+1}\left(D_{a+; g}^{(n+1) \alpha} f\right)\right)(x)= \\
&=\left(I_{a+; g}^{(n+1) \alpha-2 \gamma}\left(D_{a+; g}^{(n+1) \alpha} f\right)\right)(x), \quad \forall x \in[a, b] .
\end{aligned}
$$

We have derived that

$$
\left(D_{a+; g}^{2 \gamma} f\right)(x)=\left(I_{a+; g}^{(n+1) \alpha-2 \gamma}\left(D_{a+; g}^{(n+1) \alpha} f\right)\right)(x),
$$

$\forall x \in[a, b]$, under the condition $\frac{\gamma+m}{n+1}<\alpha<1$.

Similarly to the last two theorems, it holds

Theorem 9. All as in Corollary 1 and let $\gamma>0$ with $\lceil\gamma\rceil=m$. When $\frac{m+2 \gamma}{n+1}<\alpha<1$, we obtain that

$$
\begin{gathered}
\left(D_{a+; g}^{3 \gamma} f\right)(x)=\frac{1}{\Gamma((n+1) \alpha-3 \gamma)} \times \\
\times \int_{a}^{x}(g(x)-g(t))^{(n+1) \alpha-3 \gamma-1} g^{\prime}(t)\left(D_{a+; g}^{(n+1) \alpha} f\right)(t) d t
\end{gathered}
$$

$\forall x \in[a, b]$ and $\left(D_{a+; g}^{3 \gamma} f\right) \in C([a, b], X)$.

In general, we derive the iterated left fractional derivative representation formula:

Theorem 10. All as in Corollary 1 and let $\gamma>0$ with $\lceil\gamma\rceil=m$. When $\frac{m+(k-1) \gamma}{n+1}<\alpha<1, k \in \mathbb{N}$, we obtain that

$$
\begin{gathered}
\left(D_{a+; g}^{k \gamma} f\right)(x)=\frac{1}{\Gamma((n+1) \alpha-k \gamma)} \times \\
\times \int_{a}^{x}(g(x)-g(t))^{(n+1) \alpha-k \gamma-1} g^{\prime}(t)\left(D_{a+; g}^{(n+1) \alpha} f\right)(t) d t
\end{gathered}
$$


$\forall x \in[a, b]$ and $\left(D_{a+; g}^{k \gamma} f\right) \in C([a, b], X)$.

Similarly, it holds the iterated right fractional derivative representation formula:

Theorem 11. All as in Corollary 2 and let $\gamma>0$ with $\lceil\gamma\rceil=m$. Here we assume $\frac{m+(k-1) \gamma}{n+1}<\alpha<1, k \in \mathbb{N}$. Then

$$
\begin{gathered}
\left(D_{b-; g}^{k \gamma} f\right)(x)=\frac{1}{\Gamma((n+1) \alpha-k \gamma)} \times \\
\times \int_{x}^{b}(g(t)-g(x))^{(n+1) \alpha-k \gamma-1} g^{\prime}(t)\left(D_{b-; g}^{(n+1) \alpha} f\right)(t) d t
\end{gathered}
$$

$\forall x \in[a, b]$ and $\left(D_{b-; g}^{k \gamma} f\right) \in C([a, b], X)$.

It follows a left generalized and iterated fractional Poincaré type inequality:

Theorem 12. Here all as in Theorem 10. Let $p, q>1: \frac{1}{p}+\frac{1}{q}=1$. We further assume that $(k \in \mathbb{N})$

$$
1>\alpha>\max \left(\frac{m+(k-1) \gamma}{n+1}, \frac{k \gamma q+1}{(n+1) q}\right) .
$$

Then

$$
\begin{gathered}
\left\|D_{a+; g}^{k \gamma} f\right\|_{q,[a, b]} \leqslant \\
\leqslant \frac{\left(g((b)-g(a))^{((n+1) \alpha-k \gamma-1)+\frac{1}{p}}(b-a)^{\frac{1}{q}}\right.}{\Gamma((n+1) \alpha-k \gamma)(p((n+1) \alpha-k \gamma-1)+1)^{\frac{1}{p}}}\left\|\left(D_{a+; g}^{(n+1) \alpha} f\right) \circ g^{-1}\right\|_{q,[g(a), g(b)]} .
\end{gathered}
$$

Proof. We use (7). We observe that

$$
\begin{gathered}
\left\|\left(D_{a+; g}^{k \gamma} f\right)(x)\right\| \leqslant \frac{1}{\Gamma((n+1) \alpha-k \gamma)} \times \\
\times \int_{a}^{x}(g(x)-g(t))^{(n+1) \alpha-k \gamma-1} g^{\prime}(t)\left\|\left(D_{a+; g}^{(n+1) \alpha} f\right)(t)\right\| d t= \\
=\frac{1}{\Gamma((n+1) \alpha-k \gamma)} \int_{g(a)}^{g(x)}(g(x)-z)^{(n+1) \alpha-k \gamma-1}\left\|\left(D_{a+; g}^{(n+1) \alpha} f\right)\left(g^{-1}(z)\right)\right\| d z \leqslant
\end{gathered}
$$




$$
\begin{gathered}
\leqslant \frac{1}{\Gamma((n+1) \alpha-k \gamma)}\left(\int_{g(a)}^{g(x)}(g(x)-z)^{p((n+1) \alpha-k \gamma-1)} d z\right)^{\frac{1}{p}} \times \\
\times\left(\int_{g(a)}^{g(b)}\left\|\left(D_{a+; g}^{(n+1) \alpha} f\right)\left(g^{-1}(z)\right)\right\|^{q} d z\right)^{\frac{1}{q}}= \\
=\frac{(g(x)-g(a))^{\frac{p((n+1) \alpha-k \gamma-1)+1}{p}}}{\Gamma((n+1) \alpha-k \gamma)(p((n+1) \alpha-k \gamma-1)+1)^{\frac{1}{p}}}\left\|\left(D_{a+; g}^{(n+1) \alpha} f\right) \circ g^{-1}\right\|_{q,[g(a), g(b)]}
\end{gathered}
$$

Thus we have

$$
\begin{array}{r}
\left\|\left(D_{a+; g}^{k \gamma} f\right)(x)\right\|^{q} \leqslant \frac{(g(x)-g(a))^{(p((n+1) \alpha-k \gamma-1)+1) \frac{q}{p}}}{\Gamma((n+1) \alpha-k \gamma)^{q}(p((n+1) \alpha-k \gamma-1)+1)^{\frac{q}{p}}} \times \\
\times\left\|\left(D_{a+; g}^{(n+1) \alpha} f\right) \circ g^{-1}\right\|_{q,[g(a), g(b)]}^{q} .
\end{array}
$$

Therefore it holds

$$
\begin{aligned}
& \int_{a}^{b}\left\|\left(D_{a+; g}^{k \gamma} f\right)(x)\right\|^{q} d x \leqslant \\
\leqslant & \frac{(g(x)-g(a))^{(p((n+1) \alpha-k \gamma-1)+1) \frac{q}{p}}(b-a)}{\Gamma((n+1) \alpha-k \gamma)(p((n+1) \alpha-k \gamma-1)+1)^{\frac{1}{p}}}\left\|\left(D_{a+; g}^{(n+1) \alpha} f\right) \circ g^{-1}\right\|_{q,[g(a), g(b)]}
\end{aligned}
$$

proving the claim.

It follows a right generalized and iterated fractional Poincaré type inequality:

Theorem 13. Here all as in Theorem 11. Let $p, q>1: \frac{1}{p}+\frac{1}{q}=1$. We further assume that $(k \in \mathbb{N})$

$$
1>\alpha>\max \left(\frac{m+(k-1) \gamma}{n+1}, \frac{k \gamma q+1}{(n+1) q}\right) .
$$

Then 


$$
\begin{array}{r}
\left\|D_{b-; g}^{k \gamma} f\right\|_{q,[a, b]} \leqslant \frac{(g(b)-g(a))^{((n+1) \alpha-k \gamma-1)+\frac{1}{p}}(b-a)^{\frac{1}{q}}}{\Gamma((n+1) \alpha-k \gamma)(p((n+1) \alpha-k \gamma-1)+1)^{\frac{1}{p}}} \times \\
\times\left\|\left(D_{b-; g}^{(n+1) \alpha} f\right) \circ g^{-1}\right\|_{q,[g(a t), g(b)]} .
\end{array}
$$

Proof. As similar to Theorem 12 is omitted.

Next comes a left generalized and iterated fractional Opial type inequality:

Theorem 14. All here as in Theorem 10. Let $p, q>1: \frac{1}{p}+\frac{1}{q}=1$. We further assume that $(k \in \mathbb{N})$

$$
1>\alpha>\max \left(\frac{m+(k-1) \gamma}{n+1}, \frac{k \gamma q+1}{(n+1) q}\right) .
$$

Then

$$
\begin{gathered}
\int_{g(a)}^{y}\left\|\left(\left(D_{a+; g}^{k \gamma} f\right) \circ g^{-1}\right)(w)\right\|\left\|\left(\left(D_{a+; g}^{(n+1) \alpha} f\right) \circ g^{-1}\right)(w)\right\| d w \leqslant \\
\leqslant \frac{(y-g(a))^{((n+1) \alpha-k \gamma-1)+\frac{2}{p}}}{2^{\frac{1}{q}} \Gamma((n+1) \alpha-k \gamma)[(p((n+1) \alpha-k \gamma-1)+1)(p((n+1) \alpha-k \gamma-1)+2)]^{\frac{1}{p}}} \times \\
\quad \times\left(\int_{g(a)}^{y}\left\|\left(\left(D_{a+; g}^{(n+1) \alpha} f\right) \circ g^{-1}\right)(w)\right\|^{q} d w\right)^{\frac{2}{q}}, \quad \forall y \in[g(a), g(b)] .
\end{gathered}
$$

Proof. We use (7). We observe that

$$
\begin{gathered}
\left\|\left(D_{a+; g}^{k \gamma} f\right)(x)\right\| \leqslant \frac{1}{\Gamma((n+1) \alpha-k \gamma)} \times \\
\times \int_{a}^{x}(g(x)-g(t))^{(n+1) \alpha-k \gamma-1} g^{\prime}(t)\left\|\left(D_{a+; g}^{(n+1) \alpha} f\right)(t)\right\| d t= \\
=\frac{1}{\Gamma((n+1) \alpha-k \gamma)} \int_{g(a)}^{g(x)}(g(x)-z)^{(n+1) \alpha-k \gamma-1}\left\|\left(D_{a+; g}^{(n+1) \alpha} f\right)\left(g^{-1}(z)\right)\right\| d z \leqslant
\end{gathered}
$$




$$
\begin{aligned}
& \leqslant \frac{1}{\Gamma((n+1) \alpha-k \gamma)}\left(\int_{g(a)}^{g(x)}(g(x)-z)^{p((n+1) \alpha-k \gamma-1)} d z\right)^{\frac{1}{p}} \times \\
& \quad \times\left(\int_{g(a)}^{g(x)}\left\|\left(D_{a+; g}^{(n+1) \alpha} f\right)\left(g^{-1}(z)\right)\right\|^{q} d z\right)^{\frac{1}{q}}= \\
& =\frac{1}{\Gamma((n+1) \alpha-k \gamma)} \cdot \frac{(g(x)-g(a))^{\frac{p((n+1) \alpha-k \gamma-1)+1}{p}}}{(p((n+1) \alpha-k \gamma-1)+1)^{\frac{1}{p}}} \times \\
& \quad \times\left(\int_{g(a)}^{g(x)}\left\|\left(D_{a+; g}^{(n+1) \alpha} f\right)\left(g^{-1}(z)\right)\right\|^{q} d z\right)^{\frac{1}{q}} .
\end{aligned}
$$

That is

$$
\begin{aligned}
\left\|\left(D_{a+; g}^{k \gamma} f\right)(x)\right\| \leqslant \frac{(g(x)-g(a))^{\frac{p((n+1) \alpha-k \gamma-1)+1}{p}}}{\Gamma((n+1) \alpha-k \gamma)(p((n+1) \alpha-k \gamma-1)+1)^{\frac{1}{p}}} \times \\
\quad \times\left(\int_{g(a)}^{g(x)}\left\|\left(D_{a+; g}^{(n+1) \alpha} f\right)\left(g^{-1}(z)\right)\right\|^{q} d z\right)^{\frac{1}{q}}, \quad \forall x \in[a, b] .
\end{aligned}
$$

Call

$$
y=g(x),
$$

then

$$
x=g^{-1}(y) .
$$

Hence it holds

$$
\begin{aligned}
& \left\|\left(D_{a+; g}^{k \gamma} f\right)\left(g^{-1}(y)\right)\right\| \leqslant \frac{(y-g(a))^{\frac{p((n+1) \alpha-k \gamma-1)+1}{p}}}{\Gamma((n+1) \alpha-k \gamma)(p((n+1) \alpha-k \gamma-1)+1)^{\frac{1}{p}}} \times \\
& \quad \times\left(\int_{g(a)}^{y}\left\|\left(D_{a+; g}^{(n+1) \alpha} f\right)\left(g^{-1}(z)\right)\right\|^{q} d z\right)^{\frac{1}{q}}, \quad \forall y \in[g(a), g(b)] .
\end{aligned}
$$


Call

$$
\eta(y):=\int_{g(a)}^{y}\left\|\left(D_{a+; g}^{(n+1) \alpha} f\right)\left(g^{-1}(z)\right)\right\|^{q} d z,
$$

and

$$
\eta(g(a))=0
$$

Thus

$$
\eta^{\prime}(y)=\left\|\left(D_{a+; g}^{(n+1) \alpha} f\right)\left(g^{-1}(y)\right)\right\|^{q} \geqslant 0,
$$

and

$$
\left(\eta^{\prime}(y)\right)^{\frac{1}{q}}=\left\|\left(D_{a+; g}^{(n+1) \alpha} f\right)\left(g^{-1}(y)\right)\right\| \geqslant 0,
$$

$\forall y \in[g(a), g(b)]$.

Consequently, we get

$$
\begin{gathered}
\left\|\left(D_{a+; g}^{k \gamma} f\right)\left(g^{-1}(w)\right)\right\|\left\|\left(D_{a+; g}^{(n+1) \alpha} f\right)\left(g^{-1}(w)\right)\right\| \leqslant \\
\leqslant \frac{(w-g(a))^{\frac{p((n+1) \alpha-k \gamma-1)+1}{p}}}{\Gamma((n+1) \alpha-k \gamma)(p((n+1) \alpha-k \gamma-1)+1)^{\frac{1}{p}}}\left(\eta(w) \eta^{\prime}(w)\right)^{\frac{1}{q}},
\end{gathered}
$$

$\forall w \in[g(a), g(b)]$.

Then it holds

$$
\begin{gathered}
\int_{g(a)}^{y}\left\|\left(D_{a+; g}^{k \gamma} f\right)\left(g^{-1}(w)\right)\right\|\left\|\left(D_{a+; g}^{(n+1) \alpha} f\right)\left(g^{-1}(w)\right)\right\| d w \leqslant \\
\leqslant \frac{1}{\Gamma((n+1) \alpha-k \gamma)(p((n+1) \alpha-k \gamma-1)+1)^{\frac{1}{p}}} \times \\
\quad \times \int_{g(a)}^{y}(w-g(a))^{\frac{p((n+1) \alpha-k \gamma-1)+1}{p}}\left(\eta(w) \eta^{\prime}(w)\right)^{\frac{1}{q}} d w \times \\
\leqslant \frac{1}{\Gamma((n+1) \alpha-k \gamma)(p((n+1) \alpha-k \gamma-1)+1)^{\frac{1}{p}}} \times \\
\times\left(\int_{g(a)}^{y}(w-g(a))^{(p((n+1) \alpha-k \gamma-1)+1)} d w\right)^{\frac{1}{p}}\left(\int_{g(a)}^{y} \eta(w) \eta^{\prime}(w) d w\right)^{\frac{1}{q}}=
\end{gathered}
$$




$$
\begin{aligned}
& =\frac{(y-g(a))^{\frac{p((n+1) \alpha-k \gamma-1)+2}{p}}}{\Gamma((n+1) \alpha-k \gamma)[(p((n+1) \alpha-k \gamma-1)+1)(p((n+1) \alpha-k \gamma-1)+2)]^{\frac{1}{p}}} \times \\
& \times\left(\frac{\eta^{2}(y)}{2}\right)^{\frac{1}{q}}= \\
& =\frac{(y-g(a))^{((n+1) \alpha-k \gamma-1)+\frac{2}{p}}}{\left.2^{\frac{1}{q}} \Gamma(n+1) \alpha-k \gamma\right)[(p((n+1) \alpha-k \gamma-1)+1)(p((n+1) \alpha-k \gamma-1)+2)]^{\frac{1}{p}}} \times \\
& \times\left(\int_{g(a)}^{y}\left\|\left(\left(D_{a+; g}^{(n+1) \alpha} f\right) \circ g^{-1}\right)(w)\right\|^{q} d w\right)^{\frac{2}{q}},
\end{aligned}
$$

$\forall y \in[g(a), g(b)]$, proving the claim.

Also we give a right generalized and iterated fractional Opial type inequality:

Theorem 15. All here as in Theorem 11. Let $p, q>1: \frac{1}{p}+\frac{1}{q}=1$. We further assume that $(k \in \mathbb{N})$

$$
1>\alpha>\max \left(\frac{m+(k-1) \gamma}{n+1}, \frac{k \gamma q+1}{(n+1) q}\right) .
$$

Then

$$
\begin{aligned}
& \int_{y}^{g(b)}\left\|\left(\left(D_{b-; g}^{k \gamma} f\right) \circ g^{-1}\right)(w)\right\|\left\|\left(\left(D_{b-; g}^{(n+1) \alpha} f\right) \circ g^{-1}\right)(w)\right\| d w \leqslant \\
\leqslant & \frac{(g(b)-y)^{((n+1) \alpha-k \gamma-1)+\frac{2}{p}}}{\left.2^{\frac{1}{q}} \Gamma(n+1) \alpha-k \gamma\right)[(p((n+1) \alpha-k \gamma-1)+1)(p((n+1) \alpha-k \gamma-1)+2)]^{\frac{1}{p}}} \times \\
& \times\left(\int_{y}^{g(b)}\left\|\left(\left(D_{b-; g}^{(n+1) \alpha} f\right) \circ g^{-1}\right)(w)\right\|^{q} d w\right)^{\frac{2}{q}}, \quad \forall y \in[g(a), g(b)] .
\end{aligned}
$$

Proof. It is omitted as similar to Theorem 14.

Next we present a left generalized and iterated fractional HilbertPachpatte type inequality: 
Theorem 16. Here $i=1,2$. Let $a_{i}, b_{i} \in \mathbb{R}, a_{i}<b_{i}, 0<\alpha_{i}<1$, as in (8), (9), and $g_{i} \in C^{1}\left(\left[a_{i}, b_{i}\right]\right)$ that are strictly increasing, $f_{i} \in C^{1}\left(\left[a_{i}, b_{i}\right], X\right)$, $g_{i}^{-1} \in C^{1}\left(\left[g_{i}\left(a_{i}\right), g_{i}\left(b_{i}\right)\right]\right)$. Assume that $D_{a_{i}+; g_{i}}^{k_{i} \alpha_{i}} f_{i} \in C^{1}\left(\left[a_{i}, b_{i}\right], X\right)$, $k_{i}=1, \ldots, n_{i}$, and $\left(D_{a_{i}+; g_{i}}^{\lambda_{i} \alpha_{i}} f_{i}\right)\left(a_{i}\right)=0, \lambda_{i}=0,2,3, \ldots, n_{i}$, where $n_{i} \in \mathbb{N}$. Let $\gamma_{i}>0$ with $\left\lceil\gamma_{i}\right\rceil=m_{i}, k_{i} \in \mathbb{N}, p, q>1: \frac{1}{p}+\frac{1}{q}=1$. We further assume:

$$
1>\alpha_{1}>\max \left(\frac{m_{1}+\left(k_{1}-1\right) \gamma_{1}}{n_{1}+1}, \frac{k_{1} \gamma_{1} q+1}{\left(n_{1}+1\right) q}\right),
$$

and

$$
1>\alpha_{2}>\max \left(\frac{m_{2}+\left(k_{2}-1\right) \gamma_{2}}{n_{2}+1}, \frac{k_{2} \gamma_{2} p+1}{\left(n_{2}+1\right) p}\right) .
$$

Then

$$
\begin{aligned}
& \int_{g_{1}\left(a_{1}\right)}^{g_{1}\left(b_{1}\right)} \int_{g_{2}\left(a_{2}\right)}^{g_{2}\left(b_{2}\right)}\left(\left\|\left(D_{a_{1}+; g_{1}}^{k_{1} \gamma_{1}} f_{1}\right)\left(g_{1}^{-1}\left(y_{1}\right)\right)\right\|\left\|\left(D_{a_{2}+; g_{2}}^{k_{2} \gamma_{2}} f_{2}\right)\left(g_{2}^{-1}\left(y_{2}\right)\right)\right\| /\right. \\
& \left./\left[\frac{\left(y_{1}-g_{1}\left(a_{1}\right)\right)^{p\left(\left(n_{1}+1\right) \alpha_{1}-k_{1} \gamma_{1}-1\right)+1}}{p\left(p\left(\left(n_{1}+1\right) \alpha_{1}-k_{1} \gamma_{1}-1\right)+1\right)}+\frac{\left(y_{2}-g_{2}\left(a_{2}\right)\right)^{q\left(\left(n_{2}+1\right) \alpha_{2}-k_{2} \gamma_{2}-1\right)+1}}{q\left(q\left(\left(n_{2}+1\right) \alpha_{2}-k_{2} \gamma_{2}-1\right)+1\right)}\right]\right) d y_{1} d y_{2} \\
& \leqslant \frac{\left(g_{1}\left(b_{1}\right)-g_{1}\left(a_{1}\right)\right)\left(g_{2}\left(b_{2}\right)-g_{2}\left(a_{2}\right)\right)}{\Gamma\left(\left(n_{1}+1\right) \alpha_{1}-k_{1} \gamma_{1}\right) \Gamma\left(\left(n_{2}+1\right) \alpha_{2}-k_{2} \gamma_{2}\right)} \times \\
& \times\left(\int_{g_{1}\left(a_{1}\right)}^{g_{1}\left(b_{1}\right)}\left\|\left(D_{a_{1}+; g_{1}}^{\left(n_{1}+1\right) \alpha_{1}} f_{1}\right)\left(g_{1}^{-1}\left(z_{1}\right)\right)\right\|^{q} d z_{1}\right)^{\frac{1}{q}} \times \\
& \times\left(\int_{g_{2}\left(a_{2}\right)}^{g_{2}\left(b_{2}\right)}\left\|\left(D_{a_{2}+; g_{2}}^{\left(n_{2}+1\right) \alpha_{2}} f_{2}\right)\left(g_{2}^{-1}\left(z_{2}\right)\right)\right\|^{p} d z_{2}\right)^{\frac{1}{p}} .
\end{aligned}
$$

Proof. As in the proof of Theorem 14, we obtain:

$$
\begin{gathered}
\left\|\left(D_{a_{1}+; g_{1}}^{k_{1} \gamma_{1}} f_{1}\right)\left(g_{1}^{-1}\left(y_{1}\right)\right)\right\| \leqslant \\
\leqslant \frac{\left(y_{1}-g_{1}\left(a_{1}\right)\right)^{\frac{p\left(\left(n_{1}+1\right) \alpha_{1}-k_{1} \gamma_{1}-1\right)+1}{p}}}{\Gamma\left(\left(n_{1}+1\right) \alpha_{1}-k_{1} \gamma_{1}\right)\left(p\left(\left(n_{1}+1\right) \alpha_{1}-k_{1} \gamma_{1}-1\right)+1\right)^{\frac{1}{p}}} \times \\
\times\left(\int_{g_{1}\left(a_{1}\right)}^{y_{1}}\left\|\left(D_{a_{1}+; g_{1}}^{\left(n_{1}+1\right) \alpha_{1}} f_{1}\right)\left(g_{1}^{-1}\left(z_{1}\right)\right)\right\|^{q} d z_{1}\right)^{\frac{1}{q}},
\end{gathered}
$$


$\forall y_{1} \in\left[g_{1}\left(a_{1}\right), g_{1}\left(b_{1}\right)\right]$.

Also we obtain:

$$
\begin{gathered}
\left\|\left(D_{a_{2}+; g_{2}}^{k_{2} \gamma_{2}} f_{2}\right)\left(g_{2}^{-1}\left(y_{2}\right)\right)\right\| \leqslant \\
\leqslant \frac{\left(y_{2}-g_{2}\left(a_{2}\right)\right) \frac{q\left(\left(n_{2}+1\right) \alpha_{2}-k_{2} \gamma_{2}-1\right)+1}{q}}{\Gamma\left(\left(n_{2}+1\right) \alpha_{2}-k_{2} \gamma_{2}\right)\left(q\left(\left(n_{2}+1\right) \alpha_{2}-k_{2} \gamma_{2}-1\right)+1\right)^{\frac{1}{q}}} \times \\
\times\left(\int_{g_{2}\left(a_{2}\right)}^{y_{2}}\left\|\left(D_{a_{2}+; g_{2}}^{\left(n_{2}+1\right) \alpha_{2}} f_{2}\right)\left(g_{2}^{-1}\left(z_{2}\right)\right)\right\|^{p} d z_{2}\right)^{\frac{1}{p}},
\end{gathered}
$$

$\forall y_{2} \in\left[g_{2}\left(a_{2}\right), g_{2}\left(b_{2}\right)\right]$.

Multiplying (10) and (11), we get:

$$
\begin{gathered}
\left\|\left(D_{a_{1}+; g_{1}}^{k_{1} \gamma_{1}} f_{1}\right)\left(g_{1}^{-1}\left(y_{1}\right)\right)\right\|\left\|\left(D_{a_{2}+; g_{2}}^{k_{2} \gamma_{2}} f_{2}\right)\left(g_{2}^{-1}\left(y_{2}\right)\right)\right\| \leqslant \\
\leqslant \frac{1}{\Gamma\left(\left(n_{1}+1\right) \alpha_{1}-k_{1} \gamma_{1}\right) \Gamma\left(\left(n_{2}+1\right) \alpha_{2}-k_{2} \gamma_{2}\right)} \times \\
\times\left(\frac{\left(y_{1}-g_{1}\left(a_{1}\right)\right)^{p\left(\left(n_{1}+1\right) \alpha_{1}-k_{1} \gamma_{1}-1\right)+1}}{\left(p\left(\left(n_{1}+1\right) \alpha_{1}-k_{1} \gamma_{1}-1\right)+1\right)}\right)^{\frac{1}{p}}\left(\frac{\left(y_{2}-g_{2}\left(a_{2}\right)\right)^{q\left(\left(n_{2}+1\right) \alpha_{2}-k_{2} \gamma_{2}-1\right)+1}}{\left(q\left(\left(n_{2}+1\right) \alpha_{2}-k_{2} \gamma_{2}-1\right)+1\right)}\right)^{\frac{1}{q}} \times \\
\times\left(\int_{g_{1}\left(a_{1}\right)}^{y_{1}}\left\|\left(D_{a_{1}+; g_{1}}^{\left(n_{1}+1\right) \alpha_{1}} f_{1}\right)\left(g_{1}^{-1}\left(z_{1}\right)\right)\right\|^{q} d z_{1}\right)^{\frac{1}{q}} \times \\
\times\left(\int_{g_{2}\left(a_{2}\right)}^{y_{2}}\left\|\left(D_{a_{2}+; g_{2}}^{\left(n_{2}+1\right) \alpha_{2}} f_{2}\right)\left(g_{2}^{-1}\left(z_{2}\right)\right)\right\|^{p} d z_{2}\right)^{\frac{1}{p}} \leqslant
\end{gathered}
$$

(using Young's inequality for $a, b \geqslant 0, a^{\frac{1}{p}} b^{\frac{1}{q}} \leqslant \frac{a}{p}+\frac{b}{q}$ )

$$
\begin{gathered}
\leqslant \frac{1}{\Gamma\left(\left(n_{1}+1\right) \alpha_{1}-k_{1} \gamma_{1}\right) \Gamma\left(\left(n_{2}+1\right) \alpha_{2}-k_{2} \gamma_{2}\right)} \times \\
\times\left[\left(\frac{\left(y_{1}-g_{1}\left(a_{1}\right)\right)^{p\left(\left(n_{1}+1\right) \alpha_{1}-k_{1} \gamma_{1}-1\right)+1}}{\left(p\left(\left(n_{1}+1\right) \alpha_{1}-k_{1} \gamma_{1}-1\right)+1\right)}\right)+\left(\frac{\left(y_{2}-g_{2}\left(a_{2}\right)\right)^{q\left(\left(n_{2}+1\right) \alpha_{2}-k_{2} \gamma_{2}-1\right)+1}}{\left(q\left(\left(n_{2}+1\right) \alpha_{2}-k_{2} \gamma_{2}-1\right)+1\right)}\right)\right] \times
\end{gathered}
$$




$$
\begin{aligned}
& \times\left(\int_{g_{1}\left(a_{1}\right)}^{y_{1}}\left\|\left(D_{a_{1}+; g_{1}}^{\left(n_{1}+1\right) \alpha_{1}} f_{1}\right)\left(g_{1}^{-1}\left(z_{1}\right)\right)\right\|^{q} d z_{1}\right)^{\frac{1}{q}} \times \\
& \times\left(\int_{g_{2}\left(a_{2}\right)}^{y_{2}}\left\|\left(D_{a_{2}+; g_{2}}^{\left(n_{2}+1\right) \alpha_{2}} f_{2}\right)\left(g_{2}^{-1}\left(z_{2}\right)\right)\right\|^{p} d z_{2}\right)^{\frac{1}{p}} .
\end{aligned}
$$

So far we have

$$
\begin{aligned}
& \left\|\left(D_{a_{1}+; g_{1}}^{k_{1} \gamma_{1}} f_{1}\right)\left(g_{1}^{-1}\left(y_{1}\right)\right)\right\|\left\|\left(D_{a_{2}+; g_{2}}^{k_{2} \gamma_{2}} f_{2}\right)\left(g_{2}^{-1}\left(y_{2}\right)\right)\right\| / \\
& {\left[\left(\frac{\left(y_{1}-g_{1}\left(a_{1}\right)\right)^{p\left(\left(n_{1}+1\right) \alpha_{1}-k_{1} \gamma_{1}-1\right)+1}}{\left(p\left(\left(n_{1}+1\right) \alpha_{1}-k_{1} \gamma_{1}-1\right)+1\right)}\right)+\left(\frac{\left(y_{2}-g_{2}\left(a_{2}\right)\right)^{q\left(\left(n_{2}+1\right) \alpha_{2}-k_{2} \gamma_{2}-1\right)+1}}{\left(q\left(\left(n_{2}+1\right) \alpha_{2}-k_{2} \gamma_{2}-1\right)+1\right)}\right)\right] \leqslant} \\
& \leqslant \frac{1}{\Gamma\left(\left(n_{1}+1\right) \alpha_{1}-k_{1} \gamma_{1}\right) \Gamma\left(\left(n_{2}+1\right) \alpha_{2}-k_{2} \gamma_{2}\right)} \times \\
& \times\left(\int_{g_{1}\left(a_{1}\right)}^{y_{1}}\left\|\left(D_{a_{1}+g_{1}}^{\left(n_{1}+1\right) \alpha_{1}} f_{1}\right)\left(g_{1}^{-1}\left(z_{1}\right)\right)\right\|^{q} d z_{1}\right)^{\frac{1}{q}} \times \\
& \times\left(\int_{g_{2}\left(a_{2}\right)}^{y_{2}}\left\|\left(D_{a_{2}+; g_{2}}^{\left(n_{2}+1\right) \alpha_{2}} f_{2}\right)\left(g_{2}^{-1}\left(z_{2}\right)\right)\right\|^{p} d z_{2}\right)^{\frac{1}{p}} .
\end{aligned}
$$

The denominator in (12) can be zero only when $y_{1}=g_{1}\left(a_{1}\right)$ and $y_{2}=g_{2}\left(a_{2}\right)$. Therefore we obtain

$$
\begin{gathered}
\int_{g_{1}\left(a_{1}\right)}^{g_{1}\left(b_{1}\right)} \int_{g_{2}\left(a_{2}\right)}^{g_{2}\left(b_{2}\right)}\left(\left\|\left(D_{a_{1}+g_{1}}^{k_{1} \gamma_{1}} f_{1}\right)\left(g_{1}^{-1}\left(y_{1}\right)\right)\right\|\left\|\left(D_{a_{2}+; g_{2}}^{k_{2} \gamma_{2}} f_{2}\right)\left(g_{2}^{-1}\left(y_{2}\right)\right)\right\| /\right. \\
\left./\left[\frac{\left(y_{1}-g_{1}\left(a_{1}\right)\right)^{p\left(\left(n_{1}+1\right) \alpha_{1}-k_{1} \gamma_{1}-1\right)+1}}{p\left(p\left(\left(n_{1}+1\right) \alpha_{1}-k_{1} \gamma_{1}-1\right)+1\right)}+\frac{\left(y_{2}-g_{2}\left(a_{2}\right)\right)^{q\left(\left(n_{2}+1\right) \alpha_{2}-k_{2} \gamma_{2}-1\right)+1}}{q\left(q\left(\left(n_{2}+1\right) \alpha_{2}-k_{2} \gamma_{2}-1\right)+1\right)}\right]\right) d y_{1} d y_{2} \\
\leqslant \frac{1}{\Gamma\left(\left(n_{1}+1\right) \alpha_{1}-k_{1} \gamma_{1}\right) \Gamma\left(\left(n_{2}+1\right) \alpha_{2}-k_{2} \gamma_{2}\right)} \times \\
\times\left(\int_{g_{1}\left(a_{1}\right)}^{g_{1}\left(b_{1}\right)}\left(\int_{g_{1}\left(a_{1}\right)}^{y_{1}}\left\|\left(D_{a_{1}+; g_{1}}^{\left(n_{1}+1\right) \alpha_{1}} f_{1}\right)\left(g_{1}^{-1}\left(z_{1}\right)\right)\right\|^{q} d z_{1}\right)^{\frac{1}{q}} d y_{1}\right) \times
\end{gathered}
$$




$$
\begin{aligned}
& \times\left(\int_{g_{2}\left(a_{2}\right)}^{g_{2}\left(b_{2}\right)}\left(\int_{g_{2}\left(a_{2}\right)}^{y_{2}}\left\|\left(D_{a_{2}+; g_{2}}^{\left(n_{2}+1\right) \alpha_{2}} f_{2}\right)\left(g_{2}^{-1}\left(z_{2}\right)\right)\right\|^{p} d z_{2}\right)^{\frac{1}{p}} d y_{2}\right) \leqslant \\
& \leqslant \frac{1}{\Gamma\left(\left(n_{1}+1\right) \alpha_{1}-k_{1} \gamma_{1}\right) \Gamma\left(\left(n_{2}+1\right) \alpha_{2}-k_{2} \gamma_{2}\right)} \times \\
& \times\left(\int_{g_{1}\left(a_{1}\right)}^{g_{1}\left(b_{1}\right)}\left(\int_{g_{2}\left(a_{1}\right)}^{g_{1}\left(b_{2}\right)}\left\|\left(D_{a_{1}+; g_{1}}^{\left(n_{1}+1\right) \alpha_{1}} f_{1}\right)\left(g_{1}^{-1}\left(z_{1}\right)\right)\right\|^{q} d z_{1}\right)^{\frac{1}{q}} d y_{1}\right) \times \\
& \left.\times\left(\int_{g_{2}\left(b_{2}\right)}^{g_{g_{2}}\left(a_{2}\right)}\left\|\left(D_{a_{2}+; g_{2}}^{\left(n_{2}+1\right) \alpha_{2}} f_{2}\right)\left(g_{2}^{-1}\left(z_{2}\right)\right)\right\|^{p} d z_{2}\right)^{\frac{1}{p}} d y_{2}\right)= \\
& =\frac{\left(g_{1}\left(b_{1}\right)-g_{1}\left(a_{1}\right)\right)\left(g_{2}\left(b_{2}\right)-g_{2}\left(a_{2}\right)\right)}{\Gamma\left(\left(n_{1}+1\right) \alpha_{1}-k_{1} \gamma_{1}\right) \Gamma\left(\left(n_{2}+1\right) \alpha_{2}-k_{2} \gamma_{2}\right)} \times \\
& \times\left(\int_{g_{1}\left(a_{1}\right)}^{g_{1}\left(b_{1}\right)}\left\|\left(D_{a_{1}+; g_{1}}^{\left(n_{1}+1\right) \alpha_{1}} f_{1}\right)\left(g_{1}^{-1}\left(z_{1}\right)\right)\right\|^{q} d z_{1}\right)^{\frac{1}{q}} \times \\
& \times\left(\int_{g_{2}\left(b_{2}\right)}\left\|\left(D_{a_{2}+; g_{2}}^{\left(n_{2}+1\right) \alpha_{2}} f_{2}\right)\left(g_{2}^{-1}\left(z_{2}\right)\right)\right\|^{p} d z_{2}\right)^{\frac{1}{p}} .
\end{aligned}
$$

The theorem is proved.

Finally we present a right generalized and iterated fractional HilbertPachpatte type inequality:

Theorem 17. Here $i=1,2$. Let $a_{i}, b_{i} \in \mathbb{R}, a_{i}<b_{i}, 0<\alpha_{i}<1$, as in (13), (14), and $g_{i} \in C^{1}\left(\left[a_{i}, b_{i}\right]\right)$ that are strictly increasing, $f_{i} \in C^{1}\left(\left[a_{i}, b_{i}\right], X\right)$, $g_{i}^{-1} \in C^{1}\left(\left[g_{i}\left(a_{i}\right), g_{i}\left(b_{i}\right)\right]\right)$. Assume that $D_{b_{i}-; g_{i}}^{k_{i} \alpha_{i}} \in C^{1}\left(\left[a_{i}, b_{i}\right], X\right)$, $k_{i}=1, \ldots, n_{i}$, and $\left(D_{b_{i}-; g_{i}}^{\lambda_{i} \alpha_{i}} f_{i}\right)\left(b_{i}\right)=0, \lambda_{i}=0,2,3, \ldots, n_{i}$, where $n_{i} \in \mathbb{N}$. Let $\gamma_{i}>0$ with $\left\lceil\gamma_{i}\right\rceil=m_{i}, k_{i} \in \mathbb{N}, p, q>1: \frac{1}{p}+\frac{1}{q}=1$. We further assume:

$$
1>\alpha_{1}>\max \left(\frac{m_{1}+\left(k_{1}-1\right) \gamma_{1}}{n_{1}+1}, \frac{k_{1} \gamma_{1} q+1}{\left(n_{1}+1\right) q}\right) \text {. }
$$

and

$$
1>\alpha_{2}>\max \left(\frac{m_{2}+\left(k_{2}-1\right) \gamma_{2}}{n_{2}+1}, \frac{k_{2} \gamma_{2} p+1}{\left(n_{2}+1\right) p}\right) .
$$


Then

$$
\begin{gathered}
\int_{g_{1}\left(a_{1}\right)}^{g_{1}\left(b_{1}\right)} \int_{g_{2}\left(a_{2}\right)}^{g_{2}\left(b_{2}\right)}\left(\left\|\left(D_{b_{1}-; g_{1}}^{k_{1} \gamma_{1}} f_{1}\right)\left(g_{1}^{-1}\left(y_{1}\right)\right)\right\|\left\|\left(D_{b_{2}-; g_{2}}^{k_{2} \gamma_{2}} f_{2}\right)\left(g_{2}^{-1}\left(y_{2}\right)\right)\right\| /\right. \\
\left./\left[\frac{\left(g_{1}\left(b_{1}\right)-y_{1}\right)}{p\left(p\left(\left(n_{1}+1\right) \alpha_{1}-k_{1} \gamma_{1}-1\right)+1\right)}+\frac{\left(g_{2}\left(b_{2}\right)-y_{2}\right)^{q}\left(\left(n_{2}+1\right) \alpha_{2}-k_{2}-k_{2} \gamma_{2}-1\right)+1}{q\left(q\left(\left(n_{2}+1\right) \alpha_{2}-k_{2} \gamma_{2}-1\right)+1\right)}\right]\right) d y_{1} d y_{2} \\
\leqslant \frac{\left(g_{1}\left(b_{1}\right)-g_{1}\left(a_{1}\right)\right)\left(g_{2}\left(b_{2}\right)-g_{2}\left(a_{2}\right)\right)}{\Gamma\left(\left(n_{1}+1\right) \alpha_{1}-k_{1} \gamma_{1}\right) \Gamma\left(\left(n_{2}+1\right) \alpha_{2}-k_{2} \gamma_{2}\right)} \times \\
\times\left(\int_{g_{1}\left(a_{1}\right)}^{g_{1}\left(b_{1}\right)}\left\|\left(D_{b_{1}-; g_{1}}^{\left(n_{1}+1\right) \alpha_{1}} f_{1}\right)\left(g_{1}^{-1}\left(z_{1}\right)\right)\right\|^{q} d z_{1}\right)^{\frac{1}{q}} \times \\
\times\left(\int_{g_{2}\left(a_{2}\right)}^{g_{2}\left(b_{2}\right)}\left\|\left(D_{b_{2}-; g_{2}}^{\left(n_{2}+1\right) \alpha_{2}} f_{2}\right)\left(g_{2}^{-1}\left(z_{2}\right)\right)\right\|^{p} d z_{2}\right)^{\frac{1}{p}}
\end{gathered}
$$

Proof. It is similar to Theorem 16, thus it is omitted.

Remark 2. Some examples for $g$ follow:

$$
\begin{aligned}
g(x) & =e^{x}, \quad x \in[a, b] \subset \mathbb{R}, \\
g(x) & =\sin x \\
g(x) & =\tan x, \\
\text { where } x & \in\left[-\frac{\pi}{2}+\varepsilon, \frac{\pi}{2}-\varepsilon\right], \text { where } \varepsilon>0 \text { small. }
\end{aligned}
$$

Indeed, the above examples of $g$ are strictly increasing and continous functions.

One can apply all of our results here for the above specific choices of g. We choose to omit this job.

\section{References}

[1] Aliprantis C. D., Border K. C. Infinite Dimensional Analysis. Springer, New York, 2006.

[2] Anastassiou G. A. Principles of General Fractional Analysis for Banach space valued functions. Bulletin of Allahabad Math. Soc., 2017, vol. 32(1), pp. $71-145$. 
[3] Anastassiou G. A. Intelligent Computations: Abstract Fractional Calculus, Inequalities, Approximations. Springer, Heidelberg, New York, 2018.

[4] Bochner integral. Encyclopedia of Mathematics. URL: http://www.encyclo pediaofmath.org/index.php?title=Bochner_integral\&oldid=38659.

[5] Kreuter M. Sobolev Space of Vector-valued functions. Ulm Univ., Master Thesis in Math., Ulm, Germany, 2015.

[6] Mikkola K. Appendix B Integration and Differentiation in Banach Spaces. http://math.aalto.fi/ kmikkola/research/thesis/contents/thesisb.pdf

[7] Mikusinski J. The Bochner integral. Academic Press, New York, 1978.

Received November 19, 2019.

In revised form, December 29, 2019.

Accepted January 9, 2020.

Published online January 21, 2020.

Department of Mathematical Sciences

University of Memphis

Memphis, TN 38152, U.S.A.

E-mail: ganastss@memphis.edu 